\title{
EVALUACIÓN DEL COMPORTAMIENTO SÍSMICO DE EDIFICACIONES DE MAMPOSTERÍA EN LOS SISMOS DE MICHOACÁN DE 1985 Y 1997
}

\author{
Mario E. Rodriguez ${ }^{1}$, Patricia Alarcón ${ }^{2}$ y Raquel Machicao ${ }^{1}$
}

\begin{abstract}
RESUMEN
Se evalúa el comportamiento estructural observado en numerosas edificaciones de mamposteria en el Estado de Michoacán durante el terremoto de Caleta de Campos, del 11 de enero de 1997. La mayor parte de estas edificaciones están destinadas a viviendas y son de uno a dos niveles. Se analizan varios registros de aceleraciones obtenidos en este evento sísmico, así como algunos del terremoto de Michoacán del 19 de septiembre de 1985, y se selecciona uno que se considera representativo del movimiento del terreno en el terremoto de Caleta de Campos. Con este registro, y empleando procedimientos aproximados de análisis elásticos e inelásticos, se estudian las demandas de resistencia y de deformaciones en estructuras de mamposteria y se comparan con sus posibles capacidades. Además, se emplea una medida de daño sísmico global propuesta en la literatura para evaluar los daños causados por los terremotos en estudio. Con base en los resultados obtenidos, se evalúan los criterios de diseño sísmico empleados por reglamentos para estructuras de mamposteria.
\end{abstract}

\begin{abstract}
This paper evaluates the structural behavior of masonry buildings, observed during the January 11, 1997, Caleta de Campos earthquake, in Michoacan, Mexico. Most of these buildings were family dwellings, of one or two stories. Several acceleration records obtained in this earthquake, as well as other records obtained in the September 19, 1985, Michoacan earthquake, are analyzed. After comparing the calculated intensities of these records, a nearfield record obtained in the Caleta de Campos earthquake is selected and it is considered representative of the ground movement in the damaged area. This record is used along with approximated linear and nonlinear analyses, in which strength and displacement demands in typical masonry buildings are compared with their capacities. The observed seismic damage in masonry buildings is also related to results using a seismic damage parameter proposed in the literature. Based on the results of this study, an evaluation of current design procedures for masonry buildings is performed.
\end{abstract} Artículo recibido el 25 de febrero de 1998 y aprobado para su publicación el 29 de diciembre de 1998. Se
aceptarán comentarios y/o discusiones al artículo hasta cinco meses después de su publicación.

1 Instituto de Ingenieria, Universidad Nacional Autónoma de México

2 Universidad Michoacana de San Nicolás de Hidalgo 


\section{INTRODUCCIÓN}

La edificación a base de mampostería generalmente es la solución más empleada para resolver el problema de la vivienda en edificios de hasta cinco niveles, tanto en México como en otros países de Latinoamérica. A pesar de que diversos reglamentos de construcción estipulan procedimientos para su análisis y diseño, lo que supone una solución racional al problema, llama la atención algunos aspectos que parecen contradictorios en estos reglamentos. Por ejemplo, en el caso de México, a pesar del buen comportamiento que en general se observó en estructuras a base de muros de mampostería confinada durante el terremoto de Michoacán del 19 de septiembre de 1985, las fuerzas sísmicas de diseño especificadas por actuales reglamentos de construcción han aumentado con respecto a las especificadas por reglamentos anteriores al terremoto mencionado. Este es el caso de las normas para la construcción de muros de mampostería de los Reglamentos de Construcciones para el Distrito Federal de 1976 y de 1993 (RCDF 76, RCDF 93), de las normas respectivas para el estado de Guerrero (Meli, 1994), o de las recomendaciones del Manual de Diseño por Sismo de la Comisión Federal de Electricidad de 1980 y 1993 (CFE 80, CFE 93).

Por otro lado, aun cuando las edificaciones de mampostería no confinada son consideradas en los criterios de reglamentos para el análisis y diseño sísmico de edificaciones (por ejemplo el RCDF 93), estos criterios no han alcanzando su objetivo de reducir a niveles aceptables la vulnerabilidad sísmica de este tipo de edificaciones. En muchos eventos sísmicos, se ha observado que este tipo de edificaciones ha experimentado cantidades y niveles de daño considerables, como en el caso del terremoto de Caleta de Campos (Michoacán), del 11 de enero de 1997, que se analiza en este trabajo. Este problema se agrava si se toma en cuenta que en gran parte de la construcción rural en México se emplean muros de mampostería no confinada y es del tipo informal.

Es urgente el llevar a la práctica procedimientos sencillos que permitan reducir la vulnerabilidad sísmica de edificaciones con muros de mampostería no confinada, así como procedimientos de análisis y diseño de edificaciones a base de muros de mampostería confinada que no lleven a diseños excesivamente conservadores y por tanto antieconómicos. Un paso necesario e importante para lograr estos objetivos es el de comparar y calibrar la respuesta sísmica en edificaciones con muros de mampostería, observada durante terremotos, con la que se obtendría con procedimientos analíticos para la evaluación de la respuesta sísmica y de daño en este tipo de edificaciones. Estudios de este tipo se han efectuado para los casos de dos edificaciones de mampostería en la ciudad de México durante el sismo del 19 de septiembre de 1985 (Flores y Alcocer, 1996).

En este trabajo se describe el comportamiento global observado en numerosas edificaciones de mampostería en el estado de Michoacán, durante el terremoto de Caleta de Campos. Se analizan las posibles demandas de resistencia y de deformación en las referidas edificaciones y se comparan con capacidades calculadas. Se efectúa también un análisis semejante para este tipo de edificaciones en la misma zona, empleando registros del terremoto de Michoacán de 1985 en la zona epicentral. Además, se emplea una 
Evaluación del comportamiento sísmico de edificaciones...

medida de daño sísmico global propuesta en la literatura para evaluar el daño sísmico en las edificaciones de mampostería estudiadas, asociado a los terremotos de Michoacán de 1985 y 1997.

\section{CARACTERÍSTICAS DE LOS TERREMOTOS DE MICHOACÁN Y CALETA DE CAMPOS Y REGISTROS SÍSMICOS ANALIZADOS}

En esta investigación se analizan los terremotos de Michoacán, del 19 de septiembre de 1985, de magnitud 8.1 (Ms), y el de Caleta de Campos, del 11 de enero de 1997, el cual tuvo una magnitud (Me) de 7.3, haciendo énfasis en este último. El epicentro del terremoto de Caleta de Campos se localizó en la costa del Estado de Michoacán, con coordenadas $18.09 \mathrm{~N}, 102.86 \mathrm{O}$, cuya localización se ilustra en la Fig. 1. Es de interés observar que la profundidad de este terremoto fue de alrededor de $30 \mathrm{~km}$ (Anderson et al, 1997), y que el epicentro se ubicó dentro de la zona de ruptura de la Placa de Cocos que provocó el mencionado terremoto de Michoacán de 1985 (Fig. 1), el cual se considera como típico de subducción. Este último terremoto se originó a una profundidad menor que $30 \mathrm{~km}$.

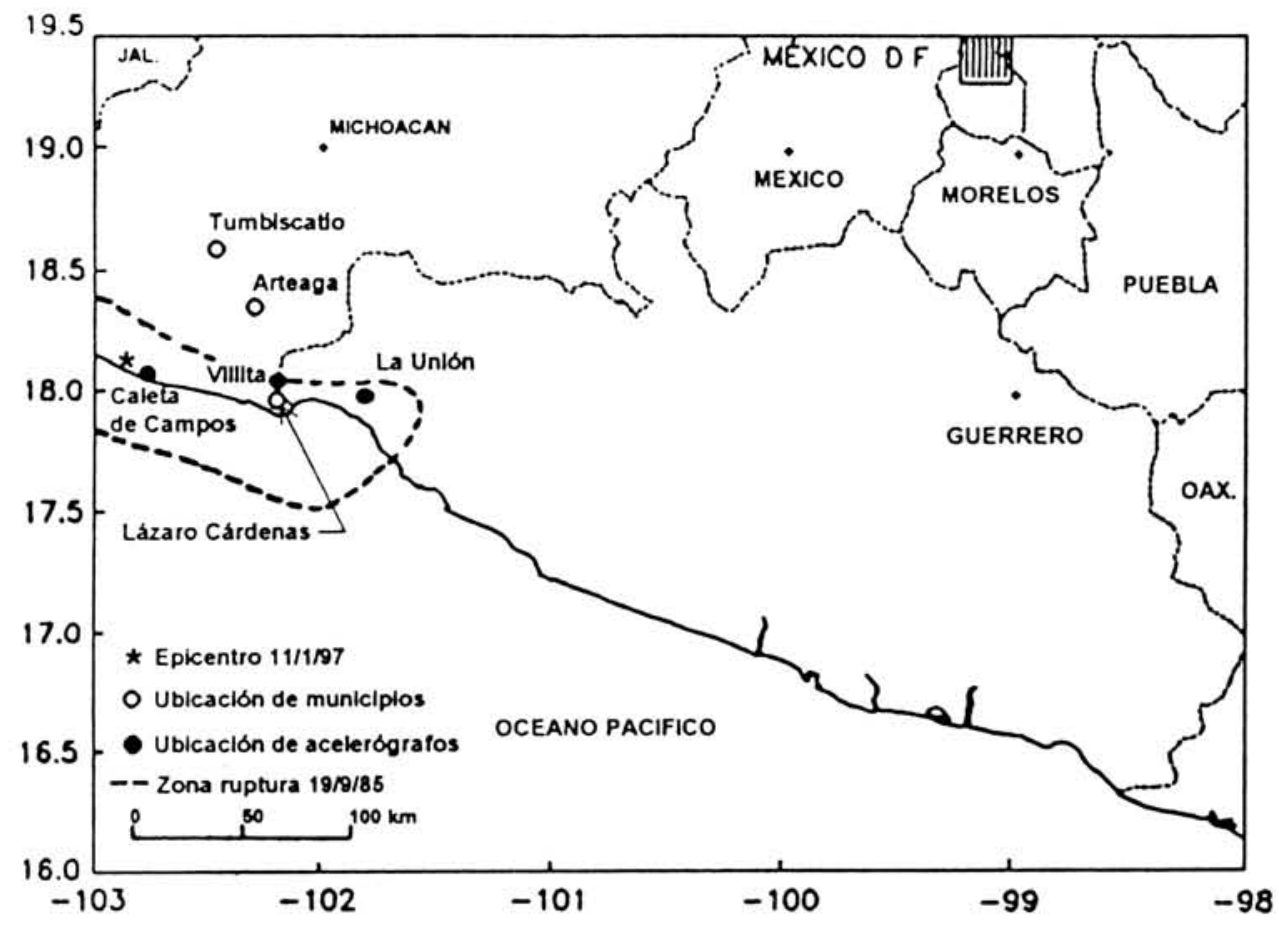

Fig 1. Ubicación de estaciones de acelerógrafos y de epicentros de los terremotos de Michoacán de 1985 y 1997

La Iabla 1 permite evaluar algunas características de registros de aceleraciones obtenidos en los terremotos de Michoacán de 1985 y de 1997. Estos registros de aceleraciones se obtuvieron en tres estaciones que se consideran de interés para evaluar la 
capacidad destructiva de los terremotos en estudio. Estas estaciones son Caleta de Campos, La Unión y Villita, cuyas ubicaciones se ilustran en la Fig. 1, en la que se aprecia además la cercanía de la estación Caleta de Campos al epicentro del terremoto de 1997. En este terremoto, las aceleraciones máximas registradas en esta estación para las componentes horizontal y vertical fueron cercanas a $0.4 \mathrm{~g}$ (Anderson et al, 1997), valor bastante mayor que las máximas registradas en esta misma estación en el terremoto del 19 de septiembre de 1985, las cuales alcanzaron el valor $0.14 \mathrm{~g}$ (Tabla 1). Por el contrario, las aceleraciones máximas obtenidas en las estaciones La Unión y Villita en el terremoto de 1997 fueron menores que las obtenidas en estas estaciones en el terremoto de 1985 (Tabla 1). El análisis del efecto de las aceleraciones verticales en la respuesta sísmica de edificaciones de mampostería no es objeto de este estudio.

Tabla 1. Características de los registros analizados

\begin{tabular}{|c|c|c|c|c|c|c|c|c|}
\hline \multirow{3}{*}{ Estación } & \multicolumn{4}{|c|}{$A_{\max } / g$} & \multicolumn{4}{|c|}{$\ln (\mathrm{m} / \mathrm{s})$} \\
\hline & \multicolumn{2}{|c|}{1997} & \multicolumn{2}{|c|}{1985} & \multicolumn{2}{|c|}{1997} & \multicolumn{2}{|c|}{1985} \\
\hline & $\mathrm{S} 90 \mathrm{E}$ & $\mathrm{SOOE}$ & S90E & SOOE & S90E & SOOE & $\mathrm{S} 90 \mathrm{E}$ & $S O O E$ \\
\hline Calcta de Canpos & 0.40 & 0.36 & 0.14 & 0.14 & 2.06 & 1.60 & 0.47 & 0.66 \\
\hline La Unión & 0.08 & 0.08 & 0.15 & 0.17 & 0.07 & 0.05 & 0.90 & 1.02 \\
\hline Villita & 0.10 & 0.11 & 0.13 & 0.13 & 0.14 & 0.09 & 0.28 & 0.42 \\
\hline
\end{tabular}

La Tabla 1 también muestra resultados de evaluar el potencial destructivo de los registros considerados, medido con la intensidad de Arias, $I_{A}$, (Arias, 1970). Con el objeto de simplificar posteriores análisis, los registros que se analizan tienen duraciones menores que las originales, lo que se hizo considerando la parte del registro con intensidades de Arias de al menos el $95 \%$ de las intensidades originales. Como se aprecia en la referida tabla, para el terremoto de 1997 los registros de Caleta de Campos tienen un potencial destructivo bastante mayor que los correspondientes a los registros de las otras estaciones consideradas. Debido a esta característica, y con el objeto de tener una estimación conservadora del movimiento del terreno, en la mayoría de los casos de edificaciones analizadas en este estudio, se seleccionó el registro Caleta de Campos, componente S90E, en sus primeros 30 segundos (Fig. 2), para analizar la respuesta de edificaciones a base de muros de mampostería. En adelante, a este registro se le identifica con la abreviatura CC97. Para fines de comparación con este registro, la Fig. 3 muestra el registro original de aceleraciones, componente S00E. obtenido en Caleta de Campos en el terremoto de 1985. En adelante este registro se identifica como CC85.

Es de interés observar que la intensidad de Arias para los registros de Caleta de Campos en 1997 son mayores que las intensidades correspondientes a los registros de 1985 obtenidos en todas las estaciones consideradas (Tabla 1). Por el contrario, de manera semejante a los casos de aceleraciones máximas anteriormente comentados, las 
intensidades de Arias para los registros obtenidos en 1997 en las estaciones La Unión y Villita son menores que las intensidades correspondientes a los registros obtenidos en estas estaciones en el terremoto de 1985 (Tabla 1).

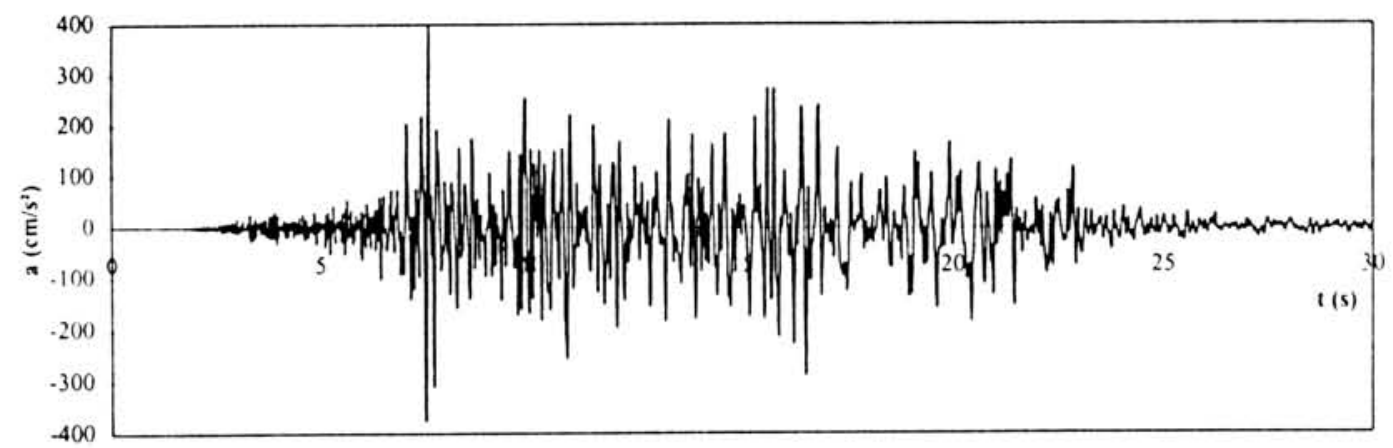

Fig 2. Registro de aceleraciones Caleta de Campos 1997, S 90 E

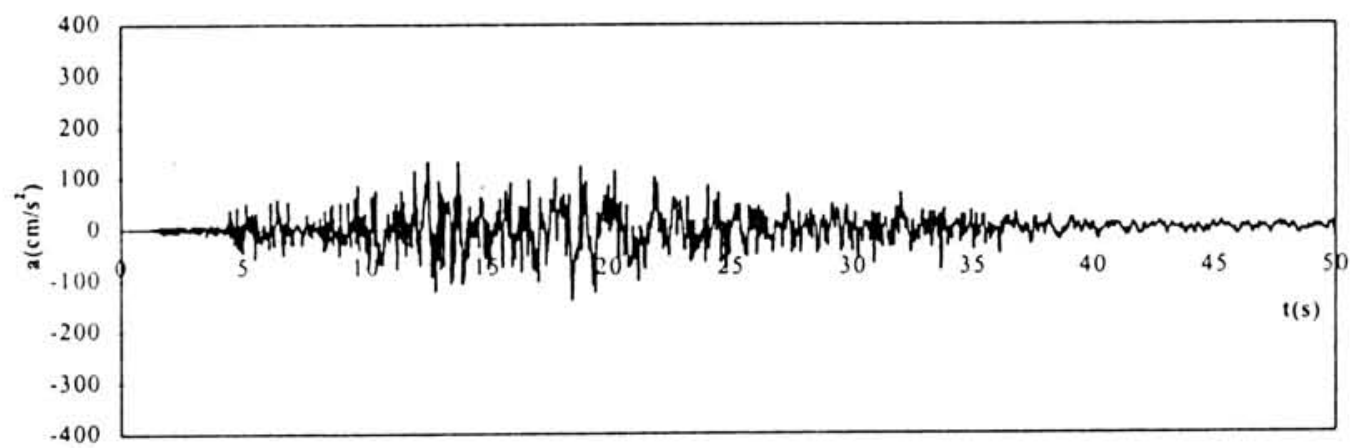

Fig 3. Registro de aceleraciones Caleta de Campos 1985, S00 E

Las características de aceleraciones máximas e intensidades de Arias anteriormente comentadas para los registros analizados en los terremotos de 1985 y 1997 pueden explicarse si se toma en cuenta los diferentes mecanismos que originaron estos terremotos. El terremoto de 1985 estuvo asociado a un mecanismo típico de subducción de la Placa de Cocos en México. Por el contrario, el mecanismo de falla del terremoto de 1997 correspondió a uno de tipo normal, asociado a deformaciones en la placa, con menos deslizamiento en ésta y por tanto con más irregularidades que podrían causar movimientos fuertes del terreno (Anderson et al, 1997).

Las Figs. 4 a la 6 muestran espectros elásticos de pseudoaceleraciones, $S_{a} / g$, para los registros de mayor intensidad (medidos con el parámetro $I_{A}$, Tabla 1), obtenidos durante los terremotos de 1985 y 1997, en las estaciones Caleta de Campos, La Unión y Villita, respectivamente. Estos registros correspondieron a las componentes S00E y S90E para los terremotos de 1985 y 1997, respectivamente. Estos espectros se obtuvieron conside- 
rando para $\xi$, factor de amortiguamiento crítico, el valor de 0.05 . Por definición, el parámetro $S_{a}$ es la resistencia por unidad de masa en una estructura de un grado de libertad ( $1 \mathrm{gdl}$ ), por lo que de manera indistinta podemos referirnos a demandas del parámetro $S_{a} \mathrm{o}$ a demandas de resistencia en la estructura. La comparación de estas respuestas muestra una tendencia semejante a la anteriormente comentada para las aceleraciones máximas e intensidades de Arias de los movimientos del estudio. Por ejemplo, considerando la estación Caleta de Campos, en estructuras con periodos menores que alrededor de $0.5 \mathrm{~s}$, las demandas de resistencia para el terremoto de 1997 son mayores que las correspondientes al terremoto de 1985 (Fig. 4). Por el contrario, los registros de las estaciones La Unión y Villita para el terremoto de 1985, excepto en intervalos de periodos cortos para el último caso, llevan a demandas de resistencia mayores que aquellas correspondientes al terremoto de 1997 (Figs. 5 y 6).

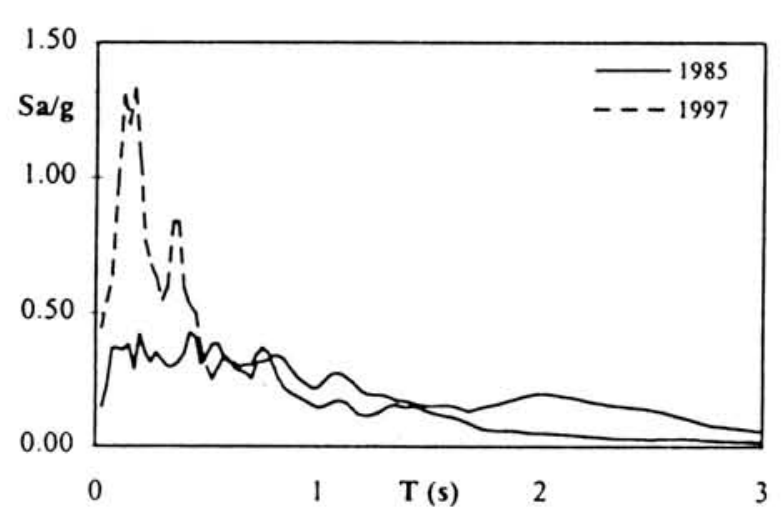

Fig 4. Demandas de resistencia elástica. Estación Caleta de Campos

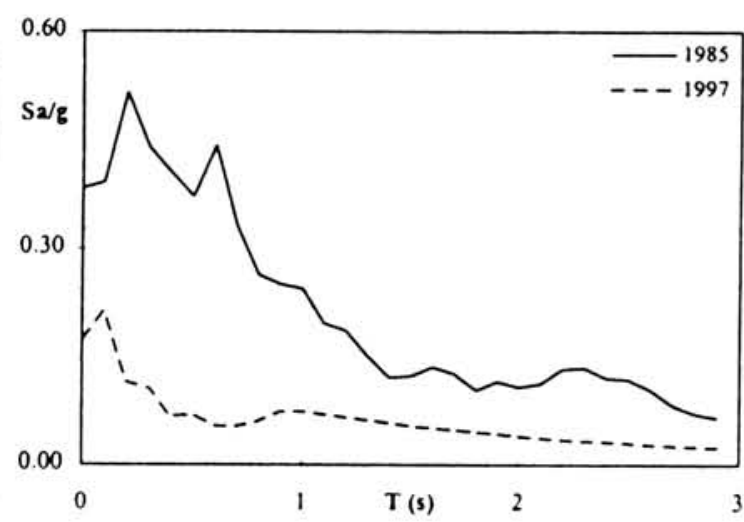

Fig 5. Demandas de resistencia elástica. Estación La Unión

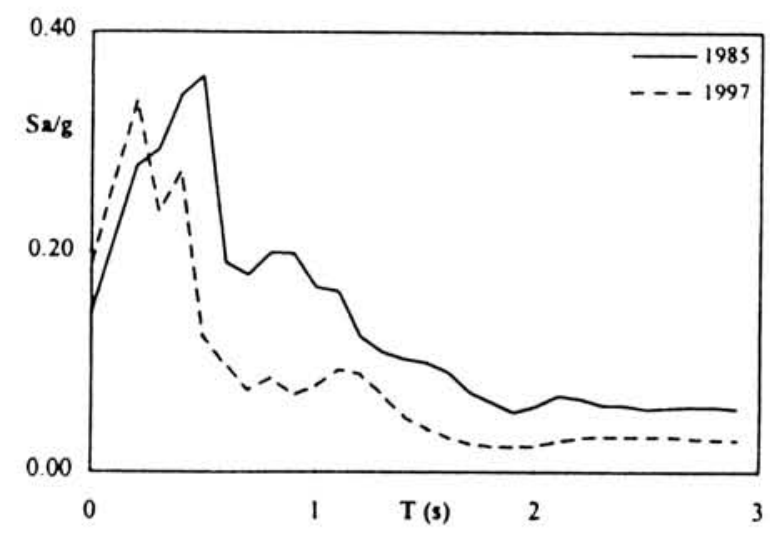

Fig 6. Demandas de resistencia elástica. Estación La Villita

Las características de las intensidades de Arias y de demandas de resistencia asociadas a los registros de aceleraciones correspondientes a los terremotos de 1985 y 1997, anteriormente comentadas, ponen en evidencia la importancia en el riesgo sísmico de estructuras, del tipo de mecanismo de falla que origina un terremoto. 
Evaluación del comportamiento sísmico de edificaciones...

\section{EVALUACIÓN DE DAÑOS EN EDIFICACIONES A BASE DE MAMPOSTERÍA EN LA ZONA EPICENTRAL DE LOS TERREMOTOS DE MICHOACÁN Y CALETA DE CAMPOS}

\section{Terremoto de Michoacán}

A pesar de que los efectos del terremoto de Michoacán de 1985 en estructuras en la ciudad de México están bien documentados, la información sobre estos efectos en la zona epicentral es bastante escasa y no existen estadísticas de daños o colapsos en esta zona (Rosenblueth y Ruiz, 1989; NZ Reconnaissance Team, 1988; Otani et al, 1995). Sin embargo, existe un consenso general de que la mayor parte de daños o colapsos en edificaciones en este terremoto ocurrió en la ciudad de México (Rosenblueth y Ruiz, 1989). La ausencia de estadísticas de daños o colapsos en edificaciones en la zona epicentral para el terremoto de Michoacán de 1985 no permite efectuar una comparación adecuada de daños sísmicos observados en este evento con resultados de procedimientos analíticos, como se hace en este estudio para el caso del terremoto de Caleta de Campos, por lo que la mencionada comparación para el caso del terremoto de Michoacán de 1985 que se efectúa en este estudio es de carácter general.

La observación de daños en edificaciones a base de mampostería en la zona epicentral de los terremotos en estudio, efectuada por los autores, sugiere que los daños en el Municipio de Arteaga en el terremoto de Caleta de Campos pueden considerarse, en general, mayores que los observados en el terremoto de Michoacán. Por el contrario, los daños causados por este terremoto, en el tipo de edificaciones en estudio, en Lázaro Cárdenas, Ixtapa y Zihuatanejo (ciudades en la zona epicentral) pueden considerarse mayores que los observados en estas ciudades en el terremoto de Caleta de Campos.

\section{Terremoto de Caleta de Campos}

Se efectuó una evaluación estructural de edificaciones en la zona afectada por el terremoto de Caleta de Campos, como respuesta a las respectivas solicitudes de revisión estructural de éstas por parte de los habitantes de las mismas. La información que se analiza en este estudio corresponde a resultados de la evaluación en campo de 3020 edificaciones de mampostería, labor que fue llevada a cabo por personal del Laboratorio de Modelos Estructurales y Ensayes Sísmicos de la Universidad Michoacana de San Nicolás de Hidalgo. Como se aprecia en la Tabla 2, la mayor parte de estas edificaciones (97\%) estaban destinadas a vivienda, estructuradas en su mayor parte a base de muros confinados de tabique o de muros de adobe. La Fig. 7 muestra un caso de daños típicos observados en viviendas de tabique confinado encontrados en el terremoto de estudio. Las edificaciones evaluadas se ubican en el estado de Michoacán, en los Municipios de Arteaga, Lázaro Cárdenas y Tumbiscatio, del total de edificaciones evaluadas, los porcentajes por municipio fueron $75 \%, 10 \%$ y $15 \%$, respectivamente. Es de interés observar que las últimas estadísticas del número de edificaciones en la zona, efectuadas por el INEGI en 1990, indican para los Municipios de Arteaga, Lázaro Cárdenas y Tumbiscatio, las cantidades de 1770,4560 y 28335 edificaciones, respectivamente. En este estudio se considera que estas cantidades podrían representar razonablemente las respectivas cantidades de edificaciones existentes durante este terremoto. 
Tabla 2. Estadísticas de daños observados en el terremoto de Caleta de Campos

\begin{tabular}{|c|c|c|c|c|c|c|c|c|c|c|}
\hline \multicolumn{2}{|c|}{$\begin{array}{c}\text { tipo de } \\
\text { edificación }\end{array}$} & \multirow{2}{*}{$\begin{array}{c}\begin{array}{c}\text { total de } \\
\text { edificaciones }\end{array} \\
\begin{array}{c}1770 \\
100\end{array}\end{array}$} & \multicolumn{2}{|c|}{$\begin{array}{c}\sin \\
\text { daño }\end{array}$} & \multicolumn{3}{|c|}{$\begin{array}{c}\text { daños } \\
\text { reparables }\end{array}$} & \multicolumn{3}{|c|}{ colapsos } \\
\hline \multirow[t]{3}{*}{ vivienda } & $\begin{array}{c}\text { adobe } \\
\%\end{array}$ & & \multicolumn{2}{|c|}{$\begin{array}{c}20 \\
1\end{array}$} & \multicolumn{3}{|c|}{$\begin{array}{c}1313 \\
74\end{array}$} & \multicolumn{3}{|c|}{$\begin{array}{c}437 \\
25\end{array}$} \\
\hline & & & $\mathrm{mc}$ & $\mathrm{cd}$ & $\mathrm{mc}$ & $\mathrm{cd}$ & $\mathrm{sc}$ & $\mathrm{mc}$ & $\mathrm{cd}$ & $\mathrm{sc}$ \\
\hline & $\begin{array}{c}\text { tabique } \\
\%\end{array}$ & $\begin{array}{l}1170 \\
100\end{array}$ & $\begin{array}{c}850 \\
73\end{array}$ & $\begin{array}{c}70 \\
6\end{array}$ & $\begin{array}{c}29 \\
2\end{array}$ & $\begin{array}{c}200 \\
17\end{array}$ & $\begin{array}{l}1 \\
0\end{array}$ & $\begin{array}{l}2 \\
0\end{array}$ & $\begin{array}{c}11 \\
1\end{array}$ & $\begin{array}{l}7 \\
1\end{array}$ \\
\hline \multirow[b]{2}{*}{ escuela } & \multirow[b]{2}{*}{$\%$} & & $\mathrm{mc}$ & $\mathrm{cd}$ & $\mathrm{mc}$ & $\mathrm{cd}$ & sc & $\mathrm{mc}$ & $\mathrm{cd}$ & $\mathrm{sc}$ \\
\hline & & $\begin{array}{c}75 \\
100\end{array}$ & $\begin{array}{l}19 \\
25\end{array}$ & $\begin{array}{l}2 \\
3\end{array}$ & $\begin{array}{l}21 \\
28\end{array}$ & $\begin{array}{l}19 \\
25\end{array}$ & $\begin{array}{l}0 \\
0\end{array}$ & $\begin{array}{l}4 \\
5\end{array}$ & $\begin{array}{l}10 \\
13\end{array}$ & $\begin{array}{l}0 \\
0\end{array}$ \\
\hline \multirow[b]{2}{*}{$\begin{array}{l}\text { iglesia } \\
\text { hospital }\end{array}$} & \multirow[b]{2}{*}{$\%$} & & \multirow{2}{*}{\multicolumn{2}{|c|}{$\begin{array}{l}0 \\
0\end{array}$}} & $\mathrm{mc}$ & $\mathrm{cd}$ & $\mathrm{sc}$ & $\mathrm{mc}$ & $\mathrm{cd}$ & $\mathrm{sc}$ \\
\hline & & $\begin{array}{c}5 \\
100\end{array}$ & & & $\begin{array}{c}2 \\
40\end{array}$ & $\begin{array}{c}2 \\
40\end{array}$ & $\begin{array}{l}0 \\
0\end{array}$ & $\begin{array}{l}0 \\
0\end{array}$ & $\begin{array}{l}0 \\
0\end{array}$ & $\begin{array}{c}1 \\
20\end{array}$ \\
\hline Total & $\%$ & $\begin{array}{c}3020 \\
100\end{array}$ & \multicolumn{2}{|c|}{$\begin{array}{c}961 \\
32\end{array}$} & \multicolumn{3}{|c|}{$\begin{array}{c}1587 \\
53\end{array}$} & \multicolumn{3}{|c|}{$\begin{array}{c}472 \\
15\end{array}$} \\
\hline
\end{tabular}

mc : mamposteria confinada

cd : confinamiento deficiente

sc : mamposteria sin confinamiento

La Tabla 2 muestra resultados de estadísticas de daños obtenidos de la evaluación efectuada en las 3020 edificaciones mencionadas, en la que además del tipo de edificación, se indican tres tipos de resultados de evaluación: sin daños, daños que se consideran reparables y colapsos. Si se considera el número total de edificaciones en la zona (según el INEGI, 1990), el número de edificaciones a base de mampostería con daños o colapsos, alcanza el $6 \%$ del total mencionado. Es relevante mencionar que, como se aprecia en la Tabla 2, alrededor del $90 \%$ de los casos de vivienda de tabique que tuvieron daños reparables o colapsos, correspondieron a mampostería con confinamiento deficiente o sin confinamiento.

El análisis de las estadísticas mencionadas es de mayor interés si de los daños o colapsos observados en los municipios anteriormente mencionados, se consideran en particular los efectos del terremoto en Arteaga, población ubicada aproximadamente a $55 \mathrm{~km}$ de la estación Caleta de Campos, Fig. 1. En este caso, el porcentaje de daños severos o colapsos en edificaciones a base de mampostería, respecto al total de edificaciones en esta población, fue alrededor del $26 \%$. Si estas edificaciones fueron diseñadas de acuerdo con reglamentos de construcción recientes, esta cantidad de daños es bastante 
alta y supera por mucho los niveles de daños esperados por reglamentos de construcción para un sismo frecuente. Además de la alta vulnerabilidad sísmica de numerosas edificaciones de adobe en la zona, así como de edificaciones de tabique en menor cantidad de casos, los daños mencionados se pueden explicar considerando dos factores. Uno es la relativa cercanía de Arteaga a la estación Caleta de Campos, donde, como se ha comentado, se obtuvieron los registros sísmicos con los mayores potenciales destructivos en la zona. Otro factor es la menor calidad de la construcción en Arteaga respecto a la observada por ejemplo en la ciudad de Lázaro Cárdenas, también con relativa cercanía al epicentro.

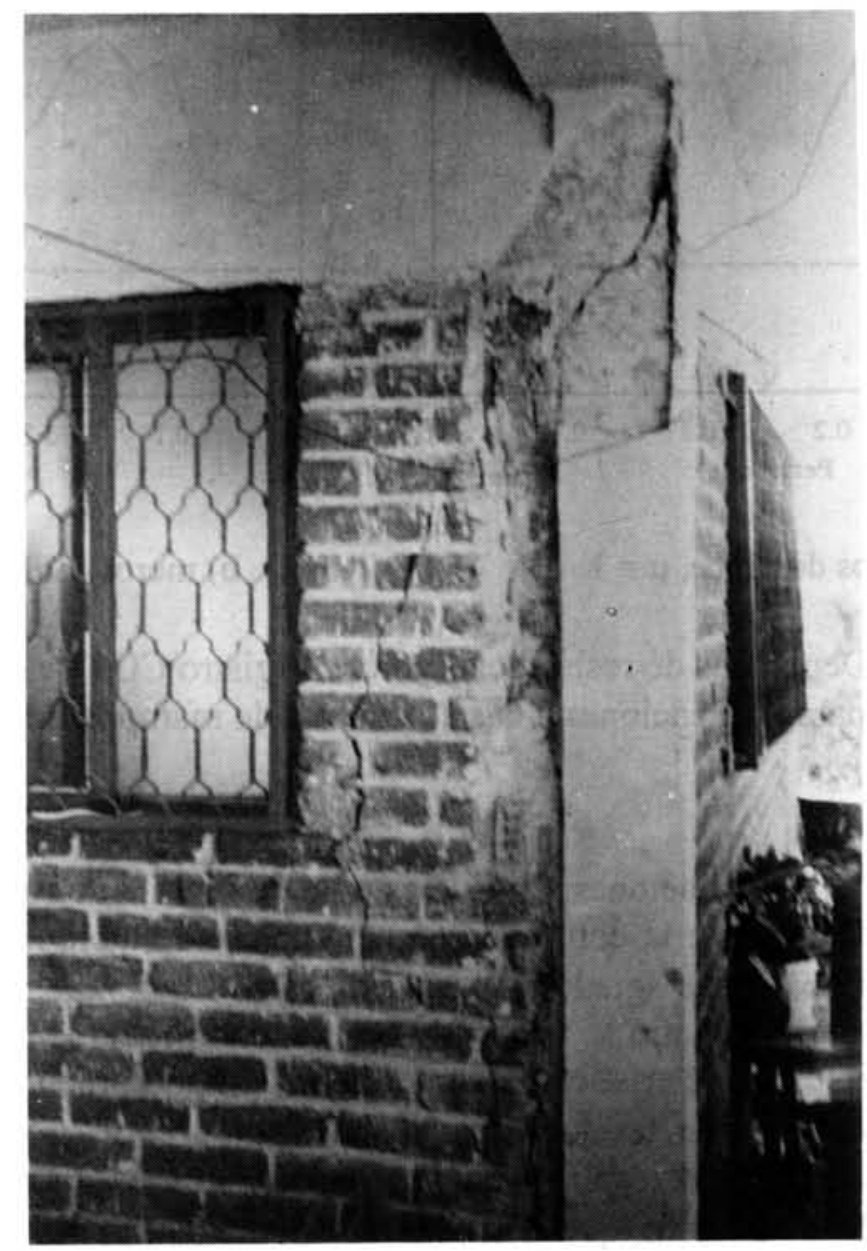

Fig 7. Daños en vivienda de tabique confinado 


\section{ANÁLISIS DE LA RESPUESTA DE EDIFICACIONES DE MAMPOSTERÍA EN EL TERREMOTO DE CALETA DE CAMPOS}

\section{Análisis de demandas y capacidades resistentes}

La Fig. 8 muestra los espectros de pseudoaceleraciones, $S_{a} / g$, correspondientes al registro CC97, considerando para $\xi$ el valor 0.05 , y para niveles de relaciones de ductilidad de desplazamientos, $\mu$, los valores uno y dos, Figs. 8 (a) y 8 (b), respectivamente. Con el propósito de efectuar comparaciones de demandas y capacidades resistentes en edificaciones a base de muros de mampostería empleando el registro CC97, así como para un

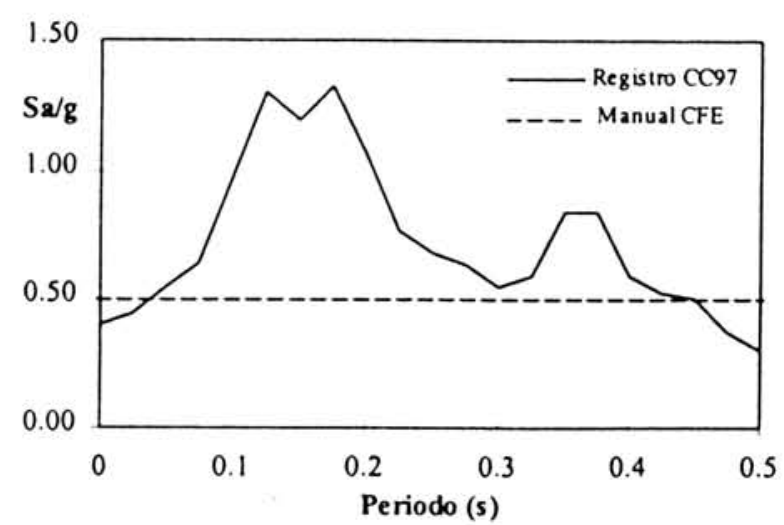

a) muros de adobe, $\mu=1$

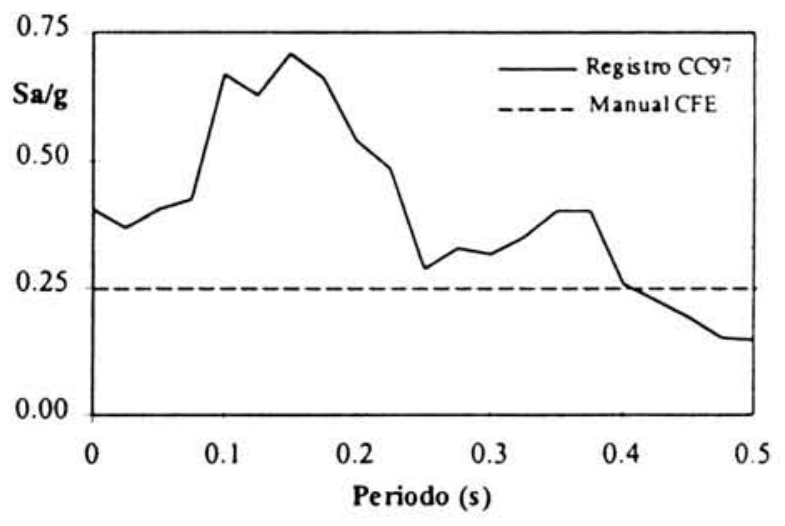

b) muros confinados, $\mu=2$

Fig 8. Demandas de resistencias para el registro CC97 y según CFE 93 para edificaciones a base de muros de mampostería

posterior análisis de deformaciones, en este estudio se considera que a las edificaciones a base de muros de mampostería confinada (generalmente de tabique macizo) se les puede asociar un valor global de $\mu$ igual a 2. Para el caso de mampostería no confinada, de tabique o adobe, en este estudio se considera sólo el comportamiento elástico de este tipo de estructuras, por sus características inherentes de comportamiento frágil, por lo que se considera para $\mu$ el valor de 1. Aun cuando la relación entre $\mu$ y el llamado factor de comportamiento sísmico que especifican los reglamentos es compleja, con un criterio simplista se puede considerar que los valores mencionados de $\mu$ también coinciden razonablemente con criterios del RCDF 93, o con los del Manual CFE 93. Dicho manual, para la zona de Michoacán, especifica demandas de resistencia en edificaciones a base de muros de mampostería que se indican en la Fig. 8, para los casos de muros confinados y sin confinar, Figs. 8 (a) y 8 (b), respectivamente. La comparación de estas demandas de resistencia, con las obtenidas con el registro CC97, indica que el Manual CFE 93 especifica demandas bastante menores que las calculadas con el registro mencionado. De manera semejante al caso de demandas de resistencia asociadas al registro SCT obtenido 
en la ciudad de México el 19 de septiembre de 1985 y las especificadas por el RCDF 93, los resultados de la Fig. 8 muestran que las recomendaciones del Manual de la CFE 93 se basan en suponer, en algunos casos, sobrerresistencias de más del doble respecto a las especificadas por este Manual. Algunos resultados experimentales en muros de mampostería confinada indican sobrerresistencias de este orden, otros resultados indican variaciones importantes en este parámetro (Ruiz, 1995; Rodríguez y Rodríguez, 1998), lo que sugiere la revisión de los criterios existentes de diseño sísmico basados en el concepto de resistencia.

Un parámetro útil para el análisis del comportamiento sísmico de estructuras a base de muros (de concreto o de mampostería) es el índice de densidad de muros, $p$, el cual en el caso de muros de mampostería, es la relación entre el área de éstos en la dirección del análisis, $A_{m}$, y el área de la planta, $A_{p}$. Con los siguientes criterios, basados en principios básicos del diseño sismorresistente, y empleados por diversos reglamentos de diseño sísmico, es posible emplear el parámetro $p$ como una medida de la resistencia sísmica mínima necesaria en una edificación. La fuerza cortante resistente global en edificios a base de muros de mampostería, $V_{R}$, se puede evaluar como:

$$
V_{R}=v_{m} A_{m}
$$

donde $v_{m}$ es el esfuerzo cortante resistente en muros de mampostería. Además, la fuerza cortante basal actuante, $V_{a c t}$, en una edificación de $n$ niveles, se puede evaluar de manera aproximada como:

$$
V_{a c t}=\frac{S_{a}}{g} n \omega^{*} A_{p}
$$

donde $\omega^{*}$ es el peso por unidad de superficie en una planta típica.

Igualando las dos expresiones anteriores se obtiene:

$$
p=\begin{array}{cc}
S_{a} & n \omega^{*} \\
g & v_{m}
\end{array}
$$

En la expresión anterior, el parámetro $p$ representa el índice de densidad de muros asociado a la capacidad resistente sísmica mínima necesaria.

De acuerdo con el RDF 93, la expresión para $v_{m}$, sin considerar los factores respectivos de reducción de resistencia, $F_{R}$, está dada por:

$$
v_{m}=\left(0.5 v^{*}+0.3 \sigma\right) \leq 1.5 v^{*}
$$

donde $v^{*}$ es el esfuerzo cortante resistente de diseño en muros de mampostería, el cual, como se comenta más adelante, para el caso de muros confinados se está considerando el valor de $3 \mathrm{~kg} / \mathrm{cm}^{2}$. El parámetro $\sigma$ es el esfuerzo vertical que actúa sobre el muro, el cual se puede evaluar como: 


$$
\sigma=\frac{W_{T}}{A_{m}}
$$

En el caso de la evaluación de las cortantes en la base de una estructura, $W_{T}$ es el peso total del edificio. Aún cuando $\sigma$ depende a su vez de la densidad de muros en la estructura, en este estudio se considera que una estimación razonable de este parámetro $\left(\right.$ en $\mathrm{kg} / \mathrm{cm}^{2}$ ) está dada por:

$$
\sigma=1.25 n
$$

Empleando la ec (5), la definición de $p$ y el valor de $\omega^{*}$ seleccionado en este estudio, es posible demostrar que en edificaciones de mampostería regulares, el valor de $p$ asociado a la ec (6) es 0.06 .

La Fig. 9 muestra resultados de emplear las ecs. (3), (4) y (6) con los valores de la relación $S_{a} / g$ que se muestran en la Fig. 8. En estos resultados para $\omega^{*}$ se consideró el valor de $800 \mathrm{~kg} / \mathrm{m}^{2}$, lo cual proviene de estimar de manera conservadora el peso propio, carga muerta y carga viva en edificaciones de mampostería. En una segunda escala de abcisas de la Fig. 9 se indica el número de niveles, $n$, asociado a los periodos fundamentales correspondientes, $T$. La relación que se consideró entre estos parámetros fue la siguiente:

$$
\mathrm{T}=\frac{n}{\lambda}
$$

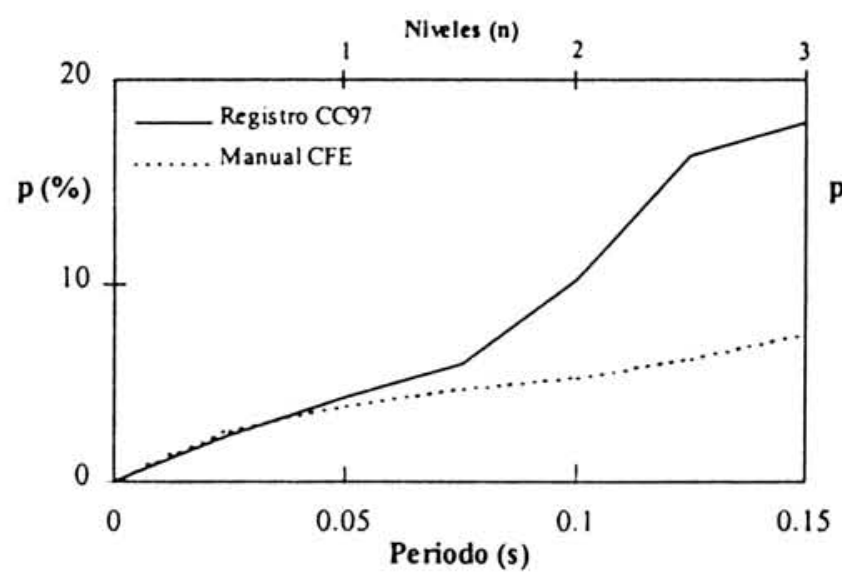

(a) Muros de adobe

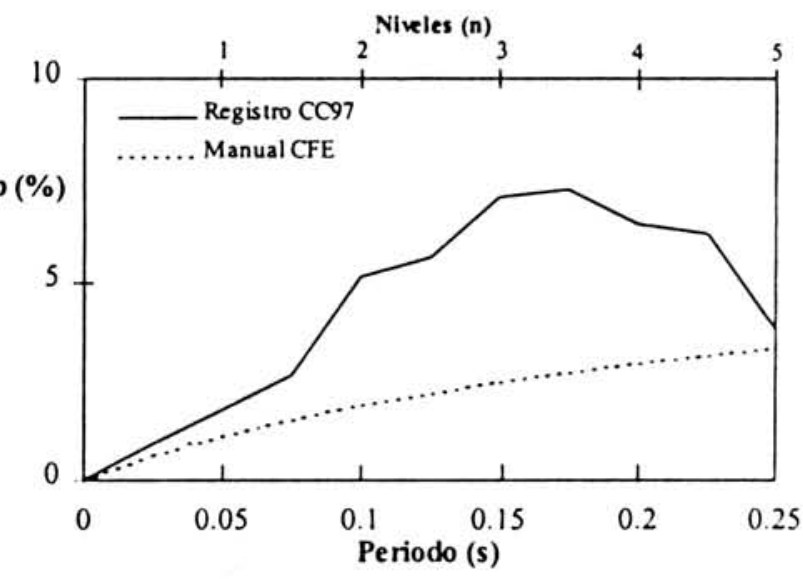

(b) Muros confinados

Fig 9. Evaluación de índices de densidad de muros en edificaciones de mampostería 
El parámetro $\lambda$ depende del tipo de estructura, tipo de excitación, densidad de muros, materiales, etc. Es de interés observar que el parámetro $p$ tiene relevancia en la evaluación de $\lambda$ (Wallace y Moehle, 1992). Por consiguiente, se debe considerar que la selección en esta investigación de un valor específico para $\lambda$ en edificaciones a base de muros de mampostería, tiene el objetivo de efectuar un análisis global aproximado de la respuesta sísmica de ellas. Resultados de periodos medidos en construcciones típicas a base de muros de mampostería, desplantados en suelo blando de la ciudad de México, indican para $\lambda$ un valor aproximadamente igual a 14 (Murià, 1991; Durán y Miranda, 1995), lo que sugiere que la selección de un valor de 20 para $\lambda$ puede ser una aproximación razonable para este tipo de construcciones en suelo firme, por lo que para estos casos en este estudio se consideró para $\lambda$ el referido valor.

Para el análisis de resistencia de edificaciones a base de muros de adobe, Fig. 9 (a), se consideró para $n$ valores representativos de uno y dos, aún cuando como referencia también se incluye el valor de tres. Además, tomando en cuenta resultados de ensayes ante cargas laterales cíclicas reversibles de dos muros de adobe estudiados en una investigación anterior en México, se consideró para $v^{*}$ el valor de $0.8 \mathrm{~kg} / \mathrm{cm}^{2}$ (Hernández et al, 1981). Se debe reconocer la alta variabilidad en la resistencia de muros de adobe, debido a la importante influencia en esta resistencia de las características no sólo de los materiales, sino también de las condiciones de humedad y temperatura, así como incluso de las características de los ensayes que se efectúan con este tipo de mampostería. Es de interés que en ensayes efectuados en Perú se obtuvieron resistencias bastante menores que la considerada en este estudio, a pesar de la semejanza en las propiedades de los adobes, lo que se pudo explicar considerando las diferencias en los morteros utilizados (Hernández et $a l, 1981)$. Esta variabilidad de la resistencia de muros de adobe se debe reconocer en la interpretación de los resultados de este estudio, por lo que éstos se deben emplear para una evaluación global y no detallada de las estructuras analizadas.

En el caso de muros confinados, Fig. 9 (b), se consideró para $n$ el valor máximo de cinco, y para $v^{*}$ el valor de $3 \mathrm{~kg} / \mathrm{cm}^{2}$. Este valor representa un límite inferior a los resultados encontrados en ensayes de muretes de tabique producidos en la zona en estudio (Alarcón y Alcocer, 1996).

Por la manera como se ha definido $p$ en la ec. (3), la comparación de este parámetro para los casos mostrados en la Fig. 9, registro CC97 y Manual CFE 93, lleva a las mismas conclusiones anteriormente comentadas para el análisis de las demandas $S_{a} / g$, Fig. 8. Sin embargo, una aplicación de interés de los resultados mostrados en la Fig. 9 es que éstos se pueden comparar con algunos valores observados en edificaciones en la zona de daños, que podrían ser típicos del parámetro $p$. Por ejemplo, es de interés observar los resultados de la evaluación detallada de la seguridad estructural efectuada en el municipio de Arteaga en seis escuelas de un nivel a base de muros de tabique confinado. En la mayoría de estas escuelas los daños fueron severos. La densidad de muros, $p$, en estos casos tuvo un promedio de 0.02 , con valores mínimos y máximos de 0.013 y 0.031 , respectivamente. 
Como se aprecia en la Fig. 9 (b), las resistencias mínimas asociadas al parámetro $p$ correspondientes al Manual CFE 93 y al registro CC97 para el caso de un nivel son aproximadamente 0.01 y 0.02 , respectivamente. Esto sugiere que en algunos casos en los que no se consideren sobrerresistencias, las densidades de muros existentes en las escuelas en estudio no satisfacían los requerimientos de resistencias mínimas necesarias asociadas al registro CC97.

\section{Análisis de demandas y capacidades de deformaciones}

Es conocido que en general los criterios de diseño sísmico de reglamentos de construcción se basan principalmente en la comparación de demandas y capacidades resistentes. Además, también se efectúa la revisión de demandas y capacidades de deformación en la estructura, lo que generalmente se hace de manera simplista y como un último paso del proceso de diseño. En el caso particular de las edificaciones a base de muros de mampostería, este paso del diseño no se efectúa, ya que los reglamentos consideran de manera implícita que este tipo de edificaciones son bastante rígidas y no deberían tener problemas de deformaciones, hipótesis que no siempre es cierta, como se analiza a continuación.

La Fig. 10 muestra resultados de evaluar espectros de desplazamientos, $S_{d}$, para el registro CC97, empleando para $\mu$ los valores de uno y dos, y para $\xi$ el valor 0.05 . Aún cuando este tipo de resultados es útil para comparar respuestas en estructuras de $1 \mathrm{gdl}$, no representa una medida directa de los probables desplazamientos relativos en la estructura. En este estudio, se considera que estos desplazamientos están representados por el llamado desplazamiento relativo global máximo, $D_{r m}$, el cual se define como:

$$
D_{r m}=\frac{\delta_{m}}{H}
$$

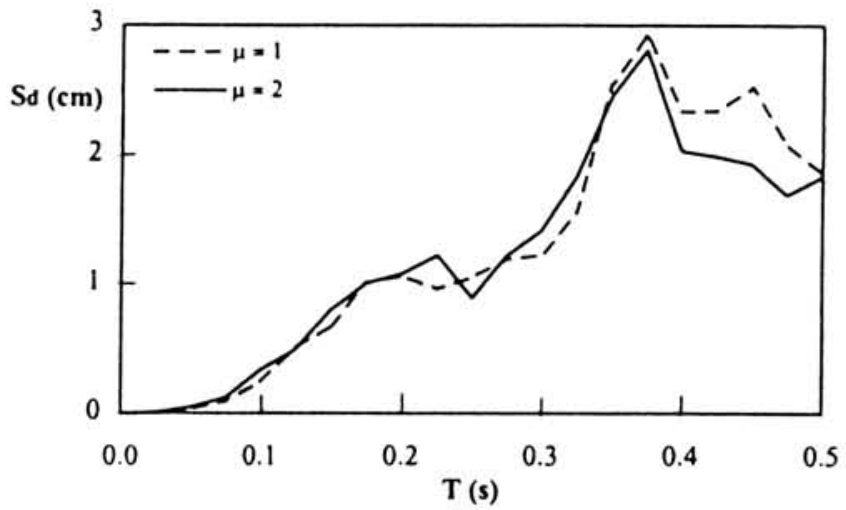

Fig 10. Espectros de desplazamiento, $\mathrm{S}_{\mathrm{d}}$, para el registro CC97 
En la expresión anterior, $\delta_{m}$ es el desplazamiento máximo del último nivel de una edificación relativo a su base, y $H$ es la altura total de la edificación. El parámetro $\delta_{m}$ se puede evaluar mediante:

$$
\delta_{m}=\gamma S_{d}
$$

en donde $\gamma$ en el intervalo elástico es el factor de participación modal. En estructuras a base de marcos regulares de varios niveles, una aproximación razonablemente conservadora de $\gamma$ en el intervalo inelástico es el valor 1.3 (Moehle, 1992; Sozen, 1997).

Mediante el empleo de (7) en edificaciones regulares, y considerando para éstas una altura de entrepiso constante, $h$, el parámetro $H$ se puede expresar como (Rodriguez, 1994):

$$
H=\frac{\alpha}{\omega}
$$

En la expresión anterior, $\omega$ es la frecuencia circular fundamental de la estructura y el parámetro $\alpha$ se define como:

$$
\alpha=2 \pi \lambda h
$$

Combinando las expresiones (8) a la (10) se obtiene

$$
D_{r m}=\gamma S_{d} \frac{\omega}{\alpha}
$$

Se evaluó el parámetro $D_{r m}$ empleando la ec. 12 para el caso del registro CC97, y considerando para $\gamma, \lambda$ y $h$ los valores $1.3,20$ y $250 \mathrm{~cm}$, respectivamente. Los resultados obtenidos, considerando para $\mu$ los valores de uno y dos, se muestran en las Figs. 11 (a) y 11 (b), correspondientes a los casos comentados de muros de adobe y tabique confinado, respectivamente. Además, en estas figuras se indican con líneas punteadas las posibles capacidades de deformación, para cada caso analizado. Estas capacidades de deformación se evalúan con el parámetro $D_{r d}$, el cual se define como el desplazamiento global relativo en la edificación asociado a daños sísmicos que se pueden considerar reparables. Este parámetro se puede evaluar como:

$$
D_{r d}=\frac{\delta_{d}}{H}
$$

En la expresión anterior, $\delta_{d}$ es el desplazamiento del último nivel de la edificación, relativo a su base, asociado a daños sísmicos que se pueden considerar reparables.

Con base en resultados de ensayes ante cargas laterales cíclicas reversibles de dos muros de adobe estudiados en México, para el adobe se está considerando el intervalo entre 0.0005 y 0.001 como representativo para el parámetro $D_{r d}$ (Hernández et al, 1981). Si se acepta la validez de estos valores, así como la densidad de muros implícita en la selección efectuada de un valor único para $\lambda$, los resultados de la Fig. 11 (a) indicarían que 
la demanda de deformaciones durante el terremoto de Caleta de Campos pudo haber excedido la capacidad de deformación de edificaciones a base de muros de adobe, principalmente para los casos de dos o más niveles. Esta característica, la posible variabilidad de densidad de muros en las edificaciones analizadas, así como la inherente variabilidad del parámetro $D_{r d}$ en muros de adobe (por las mismas razones anteriormente comentadas para el análisis de las capacidades resistentes en este tipo de mampostería), ayudan a explicar los daños en edificaciones a base de este tipo de muros observados en el terremoto en estudio. Como se aprecia en la Tabla 2, prácticamente todas las edificaciones de adobe evaluadas tuvieron danos o colapsos. La variabilidad anteriormente mencionada en demandas y capacidad de deformación en edificaciones a base de adobe, sugiere la necesidad de efectuar investigaciones en esta línea.

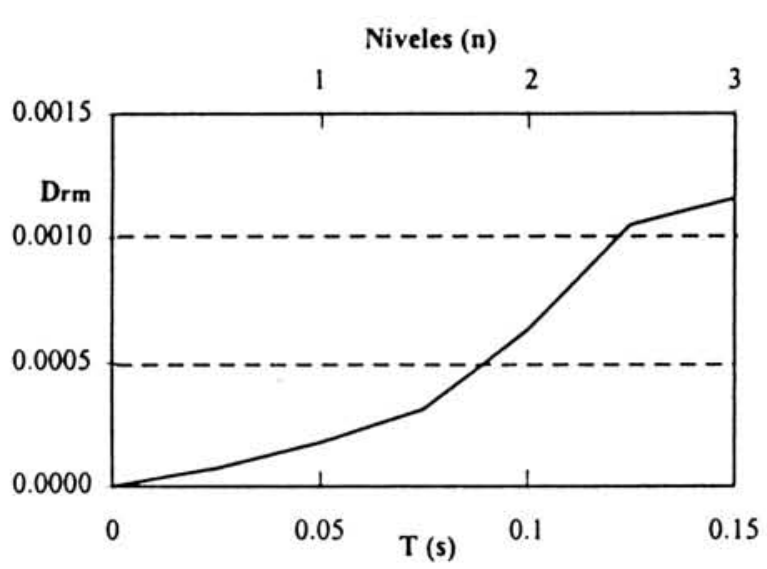

(a) Muros de adobe

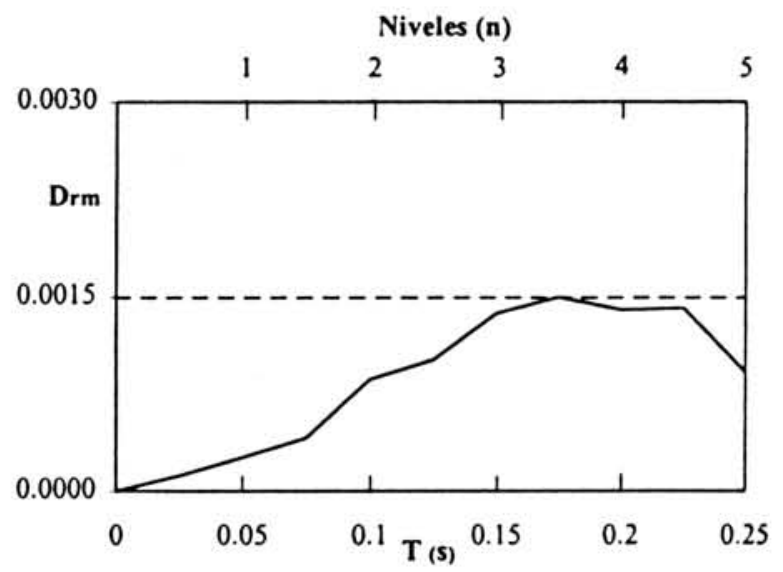

(b) Muros confinados

Fig 11. Espectros de desplazamientos, $D_{\mathrm{rm}}$. Registro CC97

Resultados de ensayes en laboratorio efectuados en México (Ruiz, 1995), empleando cargas laterales cíclicas reversibles en una edificación de dos niveles a base de muros confinados de tabique, muestran que para el valor de $D_{r m}$ igual a 0.0015 , la rigidez lateral global de la estructura se redujo al $50 \%$ de la rigidez inicial. Además, en esta estructura, cuando $D_{r m}$ alcanzó el valor 0.003 , las grietas inclinadas de la mampostería penetraron en los castillos. Es de interés mencionar que con el objeto de no superar el nivel de daños no reparables (el cual durante sismos frecuentes puede ser un nivel aceptable para el propietario), el ensaye de esta estructura finalizó para niveles de deformaciones cercanos al mencionado. Resultados comparables se encuentran en otras investigaciones (Gallegos, 1989; San Bartolomé, 1994). Con base en los resultados anteriores, en este estudio se considera para el parámetro $D_{r d}$, en muros confinados de tabique, el intervalo entre 0.0015 y 0.0025 . Con estas consideraciones, los resultados de la Fig. 11 (b) indican que las demandas de deformaciones en edificaciones de mampostería confinada, durante el 
terremoto de Caleta de Campos, pudieron haber sido cercanas al límite mencionado de 0.0015 , lo que ayudaría a explicar el nivel de daños o colapsos observados en este evento. Este nivel, expresado por ejemplo como porcentaje de la población total evaluada de viviendas a base de muros confinados, fue del $22 \%$ (Tabla 2). Es de interés mencionar que aun cuando la sobrerresistencia es un parámetro relevante en la respuesta sísmica de estructuras, el daño por sismo en ellas tiene mejor correlación con los niveles de deformaciones estructurales durante un sismo.

\section{Evaluación de una medida de daño sísmico}

En la literatura se ha propuesto el parámetro $I_{D}$, como medida del daño sísmico en edificaciones. Esta medida se define como (Rodríguez, 1994):

$$
I_{D}=\frac{\gamma^{2} E_{H}}{\left(2 \pi \lambda h D_{r d}\right)^{2}}
$$

En la expresión anterior $E_{H}$ es la energía histerética disipada al final del movimiento sísmico por una estructura de $1 \mathrm{gdl}$ con valores de $T$ y $\mu$, iguales, respectivamente, al periodo fundamental y relación máxima de ductilidad global de desplazamiento en la edificación. La Fig. 12 muestra espectros para $E_{H}$ obtenidos con el registro CC97, con valores de $\mu$ y $\xi$ iguales a 2 y 0.05 , respectivamente. Estos resultados muestran que para el registro analizado, un pequeño incremento de periodos en el intervalo de 0.1 a $0.17 \mathrm{~s}$ tiene asociado un incremento importante en las demandas de $E_{H}$. Esta característica es de interés en el análisis de $I_{D}$, ya que, de acuerdo con la ec. (14), para un tipo de estructuración específico, así como para un valor específico de $D_{r d}$, el parámetro $I_{D}$ puede considerarse proporcional a $E_{H}$ en una estructura de $1 \mathrm{gdl}$ con valores de $T$ y $\mu$ iguales, respectivamente, al periodo fundamental y relación máxima de ductilidad global de la estructura en estudio.

La Fig. 13 muestra espectros para el parámetro $I_{D}$, obtenidos con el registro CC97 y con los mismos valores de los parámetros $\gamma, \lambda$ y $h$ empleados en la evaluación de $D_{r m}$. Además, para obtener estos espectros se empleó para $\mu$ y $\xi$ los valores de 2 y 0.05 , respectivamente. Para $D_{r d d}$ se empleó el valor 0.0015 , el cual como se ha comentado, podría estar asociado a un nivel aceptable de daño sísmico en edificaciones a base de muros de mampostería confinada. Como se puede apreciar en estos resultados, la medida de daño sísmico en el tipo de edificación en estudio, para los casos de tres a cinco niveles, es bastante mayor que la de los casos de uno y dos niveles. Además, en algunos casos esta medida de daño es cercana a uno. De acuerdo con Rodríguez y Aristizabal (1998), este umbral de daño correspondería a daños severos en estructuras. Esta evaluación global de la medida de daño sísmico empleada ayuda a explicar los casos de daños severos o colapsos observados en viviendas de mampostería confinada, producidos por el terremoto en estudio (Tabla 2). 


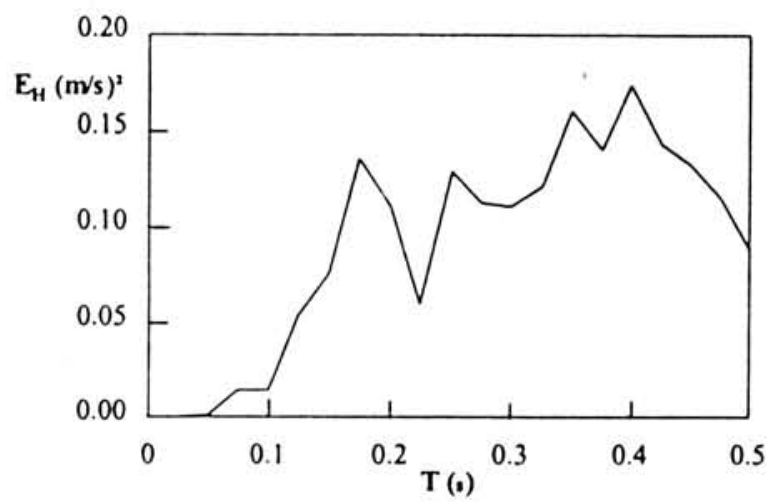

Fig 12. Espectros de energía histerética, $\mathrm{E}_{\mathrm{H}}$. Registro CC $97 ; \mu=2, \xi=0.05$

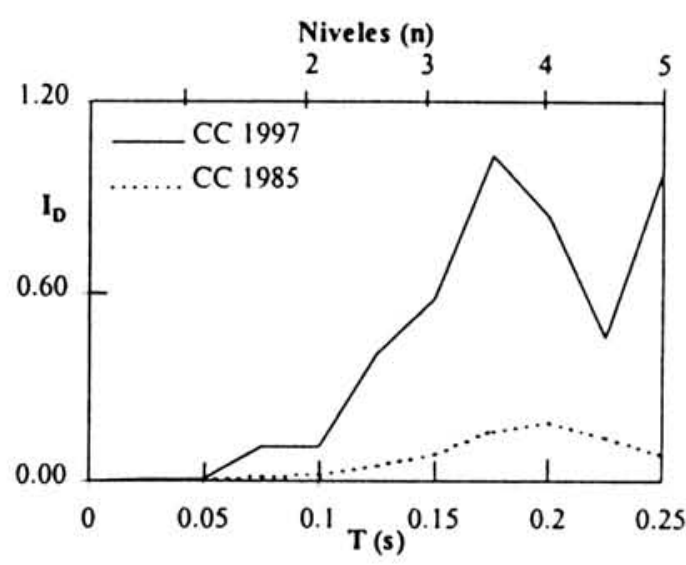

Fig 13. Medida de daño sísmico en edificaciones a base de muros de mampostería confinada. Registros CC97 y CC $85 ; \mu=2, \xi=0.05, D_{\text {rd }}=0.0015$

Es de interés analizar el posible comportamiento sísmico de las edificaciones a base de muros de mampostería que se estudian, si éstas tuvieran una capacidad de deformación mayor que la anteriormente mencionada. Por ejemplo, si como se ha comentado, consideramos el valor de 0.0025 para $D_{r d}$, la inspección de la ec 14 indica que las ordenadas de la Fig. 13 se verían afectadas por el factor $(0.0015 / 0.0025)^{2}$, es decir los valores del parámetro $I_{D}$ en esta figura se reducirían prácticamente a la tercera parte. Esto sugiere la importancia de la capacidad de deformación en muros de mampostería para reducir el daño por sismo en este tipo de estructuras, lo que se puede lograr con un confinamiento adecuado de los muros de mampostería. Con un razonamiento análogo es posible demostrar que valores menores de 0.0015 para $D_{r d}$, como podría ser el caso de mampostería de tabique sin confinar o con confinamiento deficiente, llevarían a incrementos de los valores de $I_{D}$ con respecto a los mostrados en la Fig. 13.

La Fig. 13 también muestra resultados de evaluar el parámetro $I_{D}$ para el registro CC85, considerando los mismos parámetros empleados en la evaluación de $I_{D}$ para el registro CC97 anteriormente comentados, incluyendo el valor de 0.0015 para $D_{r d}$. La comparación de valores del parámetro $I_{D}$ para los registros analizados indica que los correspondientes al registro CC85 son bastante menores que los correspondientes al registro CC97, lo cual es congruente con el mayor daño observado en edificaciones en la zona en estudio durante el terremoto de 1997, respecto a los del terremoto de 1985 .

El parámetro $I_{D}$ no es aplicable al caso de edificaciones a base de muros de adobe, ya que para éstos se está considerando un comportamiento elástico. 
Evaluación del comportamiento sísmico de edificaciones...

\section{CONCLUSIONES}

El terremoto de Caleta de Campos del 11 de enero de 1997 no causó algún tipo de daño en estructuras de la ciudad de México; sin embargo, ocasionó daños severos o colapsos en edificaciones a base de mampostería de adobe o tabique en la zona cercana al epicentro, el cual se ubicó en la zona de ruptura de la Placa de Cocos que produjo el conocido terremoto de Michoacán del 19 de septiembre de 1985. El análisis de las intensidades, medidas con la intensidad de Arias, de registros de aceleraciones obtenidos en 1997 en la estación Caleta de Campos (cercana al epicentro del evento de 1997), mostró que estos registros tuvieron intensidades bastante mayores que las de los registros de 1985 obtenidos en la zona; por el contrario, el análisis de registros obtenidos en otras estaciones en la zona, más alejadas del epicentro del evento de 1997, mostró que las intensidades de los registros de este evento fueron bastante menores que las correspondientes a los registros de 1985. Esta diferencia de intensidades, así como las diferentes características de daños o colapsos en la zona de estudio que produjeron los terremotos de 1985 y 1997, se pueden explicar si se considera que el primero fue ocasionado por el tipo de falla de subducción y el segundo por falla de tipo normal, lo que sugiere la importancia del tipo de falla en la definición del riesgo sísmico en una zona.

La evaluación estadística de daños durante el terremoto de Caleta de Campos mostró la alta vulnerabilidad de edificaciones a base de muros de adobe en la zona de estudio, así como una cantidad relevante de daños severos o colapsos en edificaciones a base de muros confinados de tabique. Estos resultados son importantes si se considera que las edificaciones de mampostería son bastante comunes en zonas rurales de México, y que el evento sísmico en estudio fue de una magnitud bastante menor que la esperada para el "sismo de diseño" y que la medida en el terremoto del 19 de septiembre de 1985.

De manera análoga al caso de demandas de resistencia especificadas por el RCDF 93 y de demandas de resistencia en estructuras en la zona de suelo blando del DF que responden al conocido registro de la SCT, las demandas de resistencia obtenidas con el registro de Caleta de Campos, en el terremoto del mismo nombre, fueron generalmente mayores que las recomendadas por el Manual de la CFE 93, lo que sugiere que estas recomendaciones implícitamente esperan en edificaciones a base de muros de mampostería, sobrerresistencias de más del doble respecto a las especificadas en este reglamento.

Las altas sobrerresistencias que se esperan en estructuras a base de muros de mampostería (en algunos casos con valores de más del doble con respecto a los obtenidos con criterios de reglamentos), la variabilidad en la capacidad resistente de muros de tabique y adobe (más en este último caso), así como el comportamiento observado en diversos terremotos en México y en el mundo en edificaciones a base de mampostería, sugieren la necesidad de revisar los criterios de diseño reglamentario para este tipo de edificaciones, las cuales se basan principalmente en la revisión de demandas y capacidades resistentes.

El análisis efectuado en esta investigación de demandas y capacidades de deformación, en edificaciones a base de muros de mampostería durante el terremoto de Caleta 
de Campos, así como el análisis de la medida de daño sísmico empleada, ayudó a explicar de manera congruente los niveles de daño y colapsos observados en estas estructuras. Aún cuando los valores empleados en este estudio para las capacidades de deformación en edificaciones de mampostería no deben considerarse como valores precisos de estas capacidades, los autores consideran que la variabilidad en éstas es menor que la correspondiente a capacidades resistentes de muros de mampostería. Además, es conocido que la demanda de deformaciones en edificaciones de mampostería durante un terremoto puede reducirse, en la mayoría de los casos, con incrementos en la densidad de muros. Estas características, de predicción de la capacidad de deformación, y de reducción en demandas de deformaciones, sugieren la necesidad de emplear un criterio alternativo de diseño sismorresistente de edificaciones de mampostería, basado principalmente en un diseño por deformaciones. Es de interés observar que este criterio es la base de recomendaciones recientes en los Estados Unidos para la rehabilitación sísmica de edificaciones existentes de diversos tipos, incluyendo las de mampostería (FEMA 273, 1997).

En este trabajo se ha empleado una medida de daño sísmico para evaluar el comportamiento sísmico de edificaciones de tabique. Esta medida emplea la energía histerética disipada por la estructura durante un terremoto, asi como una medida de la capacidad de deformación de la estructura. Los resultados de la evaluación de esta medida de daño sísmico muestra la importancia de la capacidad de deformación de las estructuras para disminuir el daño por sismo en ellas.

\section{AGRADECIMIENTO}

Los autores agradecen al Instituto de Ingeniería de la UNAM y a la Universidad Michoacana de San Nicolás de Hidalgo por las facilidades brindadas para el desarrollo de esta investigación, así como a los revisores del manuscrito por sus acertados comentarios que contribuyeron a mejorarlo.

\section{REFERENCIAS}

Alarcón, P. y Alcocer S. (1996) "Características mecánicas de la mampostería en el Estado de Michoacán, México". Memorias del VIII Curso Internacional sobre Edificaciones de Bajo Costo en Zonas Sísmicas. CISMID - JICA

Anderson, J., Quaas R., Singh S., Almora D., Velasco J., Vazquez R, Perez C., Ayala M., Castro G., Ylizaliturri J. (1997). "Accelerograms from the Guerrero, Mexico, strong motion array for the January 11, 1997 Caleta de Campos Earthquake (Mw = 7.1)", Report GAA-19, UNR Seismological Laboratory e Instituto de Ingeniería, UNAM.

Arias, A. (1970). "A measure of earthquake intensity". Seismic Design for Nuclear Power Plants (Editado por R. Hansen), Massachusetts Institute of Technology Press, Cambridge, USA, 438-483. 
Comisión Federal de Electricidad 1980 (CFE 80). "Manual de diseño de obras civiles. Diseño por sismo."

Comisión Federal de Electricidad 1993 (CFE 93). "Manual de diseño de obras civiles. Diseño por sismo."

Durán R. y Miranda E. (1995) "Demandas sísmicas en estructuras de mampostería desplantadas en suelo blando". Cuadernos de Investigación No 23, CENAPRED, 79118.

Flores, L. y Alcocer S. (1996) "Calculated response of confined masonry structures". Proc. Eleventh World Conf. on Earth. Engr., Acapulco, México, vol. 4, paper No 1830.

FEMA 273 (1997), "NEHRP Guidelines for the Seismic Rehabilitation of Buildings", Federal Emergency Management Agency, Washington D.C.

Gallegos, H. (1989) “Albañilería Estructural”, Pontificia Universidad Católica del Perú.

Hernández O., Meli R., Padilla M. y Valencia E. (1981). "Refuerzo de la vivienda económica en zonas sísmicas. Estudios experimentales". Publicación 441 de la Serie Azul del Instituto de Ingeniería de la UNAM.

Meli, R. (1994). "Mampostería estructural. La práctica, la investigación y el comportamiento sísmico observado en México", Cuadernos de Investigación, CENAPRED, No. $17,3-23$.

Moehle J. (1992) "Displacement-based design of RC Structures Subjected to Earthquakes", Earthquake Spectra Journal, vol. 8, No. 3, 403-428.

Muriá D. (1991) "Efectos de interacción suelo-estructura en edificios de mampostería". Memorias del Simposio Internacional sobre seguridad sísmica en la vivienda económica. CENAPRED, 520-542

New Zealand Reconnaissance Team (1988), "The September 1985 Mexico Earthquakes. Final Report of the New Zealand Reconnaissance Team". Bulletin of the New Zealand National Society for Earthquake Engineering, vol. 21, No. 1, 3-96.

Otani, S., Kikuchi, K., Alcocer, S. y Lopez, O. (1995). "Informe del estado actual de las edificaciones dañadas durante el sismo de Michoacán de 1985 en la zona epicentral (Revisión de los métodos de reparación y refuerzo empleados)". Cuadernos de Investigación. CENAPRED, No. 14.

Reglamento de Construcciones para el Distrito Federal 1976. (RCDF, 1976). Diario Oficial, 15 de Diciembre de 1976.

Reglamento de Construcciones para el Distrito Federal 1993. (RCDF, 1993). Gaceta Oficial del Departamento del D. F.

Rodríguez, M. (1994). 'A measure of the capacity of earthquake ground motions to damage structures'. Earthquake Eng. \& Structural Dyn. J. Vol 23, No 6, 627-643.

Rodríguez M. y Aristizábal J.C. (1998). "Evaluación de la capacidad destructiva de terremotos". Publicación de la Serie Azul del Instituto de Ingeniería, UNAM, No 609. 
Rodríguez M. y Rodríguez V. (1998) "Diseño sísmico por desempeño en edificaciones a base de mampostería". Memorias del XI Congreso Nacional de Ingeniería Estructural, Monterrey, México, 250-258.

Rosenblueth, E. y Ruiz, S. (Editores) (1989). "The Mexico Earthquake of September 19. 1985", Journal Earthquake Spectra, vol. 5, No. 1.

Ruiz, J. (1995). "Reparación y refuerzos de una estructura tridimensional de mampostería". Tesis de Maestría. División de Estudios de Posgrado, Facultad de Ingeniería, UNAM.

San Bartolomé, A. (1994) "Construcciones de albañilería - Comportamiento Sísmico y Diseño Estructural", Pontificia Universidad Católica del Perú. Fondo Editorial

Sozen M. (1997). "Drift-driven design for earthquake resistance of reinforced concrete". The EERC-CUREe Symposium in Honor of Vitelmo Bertero. Publicación UCB/EERC-97/05, 1-8

Wallace J. y Moehle J. (1992). "Ductility and detailing requirements of bearing wall buildings", Journal of Structural Engineering, ASCE, Vol. 118, No. 6, 1625-1644. 